\title{
Common mode electronic noise in differential circuits
}

\author{
Ernesto Serrano-Finetti*, Oscar Casas, Ramon Pallàs Areny \\ Instrumentation, Sensors and Interfaces Group, Universitat Politècnica de Catalunya-BarcelonaTech, c/ Esteve Terradas, 7, 08860 Castelldefels, Barcelona, Spain
}

\section{A R T I C L E I N F O}

\section{Article history:}

Received 28 December 2017

Received in revised form 26 March 2019

Accepted 1 April 2019

Available online 10 April 2019

\section{Keywords:}

Differential circuits

Instrumentation amplifiers

Electronic noise

Cross-correlation

Noise model

Common-mode rejection ratio

\begin{abstract}
A B S T R A C T
Differential circuits are assumed to reject common-mode noise yet no parameter is available to describe that rejection as the Common Mode Rejection Ratio (CMRR) applies only to deterministic signals, not to random noise. We propose a model and a method to analyze the contribution of the input common-mode electronic noise to the output voltage noise of differential circuits. The analysis shows that the same parameter-matching conditions that improve the CMRR determine common-mode noise rejection but CMRR is more sensitive to mismatching. For example, a low-frequency CMRR as low as $8 \mathrm{~dB}$ implies that more than $82 \%$ of common-mode noise is rejected. Thus, often only differential-mode noise is relevant. However, if the equivalent input current noise sources predominate over equivalent input voltage noise sources, cross-correlation between them partially yields common-mode noise that will also be rejected. We also propose a simple test that yields an estimate of that correlation.
\end{abstract}

(c) 2019 Elsevier Ltd. All rights reserved.

\section{Introduction}

Differential circuits reject input common-mode signals regardless of the origin of the signals, provided they do not exceed the specified maximal input common mode voltage. In voltageprocessing circuits, the capability of rejecting common-mode deterministic signals is described by the quotient between the voltage gain for differential signals and the voltage gain for common-mode signals, termed Common Mode Rejection Ratio (CMRR). Ideally, the CMRR of differential circuits should be large enough for input common mode signals to yield an output voltage smaller than the desired voltage resolution in the frequency range of interest.

For random signals, however, no parameter equivalent to the CMRR has been defined as the signal to noise ratio (SNR) does not differentiate common-mode noise from differential-mode noise. Instead, it is often assumed that the ability of rejecting common-mode signals does not depend on their nature hence common-mode electronic -random- noise will be rejected the same as any other common-mode signal [1-3]. The scarcity of studies about common-mode electronic noise may suggest that this assumption is widely accepted.

Most studies concerning common-mode noise do not really consider random signals but interfering signals that originate in the electromagnetic environment as in [4] or on common-mode signals that are part of the sensed measurands as in [5]. However,

\footnotetext{
* Corresponding author.

E-mail address: ernesto.serrano@upc.edu (E. Serrano-Finetti).
}

analog integrated circuits have circuit topologies where some components contribute common-mode electronic noise. For example, the tail current source of differential transistor pairs -the input stage of op amps- generates common-mode current noise. If both branches of the differential pair are completely symmetrical then identical voltage noise signals appear at each output terminal that are considered to cancel out. Breaking that symmetry converts part of the common-mode electronic noise to differential-mode [6] which is likely to happen as frequency increases. Common-mode electronic noise is also a concern in more complex circuits. In [7], the level-shifting circuitry of a switched-capacitor differential amplifier is shown to contribute common-mode electronic noise that is considered to be attenuated by the amplifier's CMRR. However, the $1 / f$ noise component of the common-mode is not sufficiently attenuated and defines the corner frequency of the differential-mode output noise spectrum. In [8], the commonmode feedback circuitry contributes common-mode electronic noise that is attenuated by a factor related to CMRR -but not the CMRR itself. In that work, it was not possible to have a more comprehensive description of that factor as no further study was carried out.

Voltage-feedback operational amplifiers (op amps) are archetype differential circuits and in the analysis of their internal noise sources, common-mode noise components are assumed not to contribute to output voltage noise because of internal subtractions and balanced differential stages [9-11]. That balance is the same that yields high CMRR. However, this does not imply that the CMRR is a valid parameter for noise analysis. 
The goal of this work is to analyze the contribution of input common-mode electronic noise to the output voltage noise in differential circuits and to find a parameter to describe that contribution. First, a model is proposed to describe how common-mode noise propagates into the signal chain inside differential circuits. Next, the cross-correlation between propagated noise signals is calculated, a parameter that describes the conditions to obtain minimal output noise, and some predictions derived from these conditions are formulated. Later on, the validity of the model and those predictions are experimentally assessed and their possible impact on the design of passive front-ends for differential circuits is discussed. Finally, since the contribution remaining of differential input noise mostly depends on the correlation between input noise currents, a simple test is proposed to estimate that correlation.

\section{Common-mode noise propagation in differential circuits}

\subsection{Circuit model and noise analysis}

Fig. 1 shows a simplified model that describes how commonmode signals propagate inside a voltage-processing circuit with differential input and single-ended output. The signal is simultaneously applied to both input terminals $(\mathrm{H}$ and $\mathrm{L})$, processed in each of the two signal paths $H_{1}(f)$ and $H_{2}(f)$ in a first stage, and the respective output voltages are subtracted in a second stage that yields a single-ended output. Eventually, later stages further process the result of the subtraction but they cannot distinguish whether that result comes from an input common-mode or differential-mode signal hence those stages are irrelevant with regard to the overall circuit performance in front of commonmode or differential-mode signals. The second stage is assumed to be ideal hence it responds only to the difference between the two signals.

If $H_{1}(f)$ and $H_{2}(f)$ are assumed to be first-order low-pass transfer functions that should ideally be equal but in practice may have different dc gains, $G_{1}$ and $G_{2}$, and bandwidths, $f_{1}$ and $f_{2}$, for example because of component manufacturing tolerance, we have,

$H_{1}(f)=\frac{G_{1} f_{1}}{f_{1}+j f}$.

$H_{2}(f)=\frac{G_{2} f_{2}}{f_{2}+j f}$.

Then, a common-mode voltage noise source with voltage spectral density (VSD) $e_{\mathrm{ni}}(f)$ will yield a noise signal with VSD $e_{\mathrm{n} 1}(f)$ at one of the outputs of the first stage and $e_{\mathrm{n} 2}(f)$ at the other output. According to [12], the cross-correlation coefficient between these two VSDs can be defined as

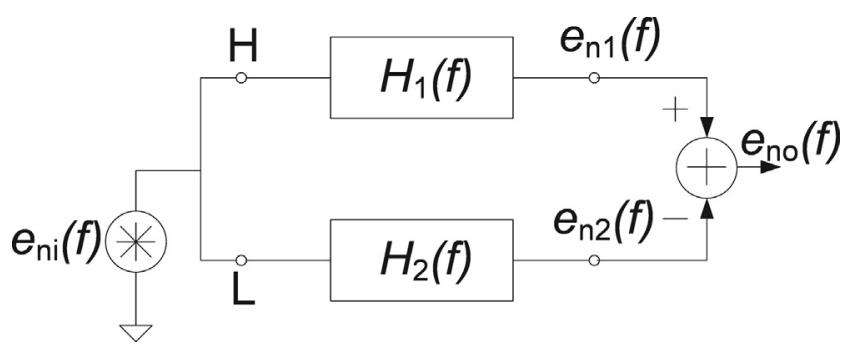

Fig. 1. A common-mode signal propagating through a differential circuit that comprises two separate transfer functions and a stage that subtracts their respective outputs to obtain a single-ended signal.
$C_{12}=\frac{R_{12}(0)}{\sigma_{1} \sigma_{2}}$

where $\sigma_{1}$ and $\sigma_{2}$ are the rms voltages due to $e_{\mathrm{n} 1}(f)$ and $e_{\mathrm{n} 2}(f)$, and $R_{12}(0)$ is their cross-correlation power. Because $e_{\mathrm{n} 1}(f)$ and $e_{\mathrm{n} 2}(f)$ are stationary random processes, $\sigma_{1}$ and $\sigma_{2}$ can be computed as

$\sigma_{x}=\sqrt{R_{x x}(0)}=E_{\mathrm{nx}}$, for $x=1,2$.

Recalling the definition of the cross-correlation function, $R_{12}(0)$ is computed as (see the Annex)

$R_{12}(0)=\frac{1}{2 \pi} \int_{-\infty}^{\infty} e_{\mathrm{n} 1}(f) e_{\mathrm{n} 2}^{*}(f) \mathrm{d} f$

and, if $e_{\mathrm{ni}}(f)$ is white,

$R_{12}(0)=\frac{e_{\mathrm{ni}}^{2}}{2 \pi} \int_{-\infty}^{\infty} H_{1}(f) H_{2}^{*}(f) \mathrm{d} f=2 \pi e_{\mathrm{ni}}^{2} G_{1} G_{2} \frac{f_{1} f_{2}}{f_{1}+f_{2}}$

Using (4) to compute $\sigma_{1}$ yields

$$
\begin{aligned}
R_{11}(0) & =\frac{1}{2 \pi} \int_{-\infty}^{\infty} e_{\mathrm{n} 1}(f) e_{\mathrm{n} 1}^{*}(f) \mathrm{d} f=\frac{e_{\mathrm{ni}}^{2}}{2 \pi} \int_{-\infty}^{\infty} \frac{f_{1}^{2} G_{1}^{2}}{f_{1}^{2}+f^{2}} \mathrm{~d} f \\
& =e_{\mathrm{ni}}^{2} G_{1}^{2} \pi f_{1} .
\end{aligned}
$$

Therefore,

$\sigma_{1}=e_{\mathrm{ni}}\left|G_{1}\right| \sqrt{\pi f_{1}}$

and, similarly,

$\sigma_{2}=e_{\mathrm{ni}}\left|G_{2}\right| \sqrt{\pi f_{2}}$

Finally, the correlation coefficient will be

$C_{12}=\frac{2 \pi e_{\mathrm{n}}^{2} G_{1} G_{2} \frac{f_{1} f_{2}}{f_{1}+f_{2}}}{e_{\mathrm{ni}}^{2}\left|G_{1}\right| \sqrt{\pi f_{1}}\left|G_{2}\right| \sqrt{\pi f_{2}}}=2 \frac{\sqrt{f_{1} f_{2}}}{f_{1}+f_{2}} \operatorname{sgn}\left(G_{1}\right) \operatorname{sgn}\left(G_{2}\right)$

that shows that spectral correlation depends on the closeness between the circuit bandwidths hence on the frequency contents of the two processed signals, but does not depend on their amplitudes. The average bandwidth $f_{\mathrm{a}}=\left(f_{1}+f_{2}\right) / 2$ and their imbalance $\Delta f=f_{1}-f_{2}$ allow us to define the bandwidth tolerance as $t_{\mathrm{f}}=2 f_{\mathrm{a}} / \Delta f$ hence $f_{1}=f_{\mathrm{a}}\left(1+t_{\mathrm{f}}\right)$ and $f_{2}=f_{\mathrm{a}}\left(1-t_{\mathrm{f}}\right)$. Therefore, $C_{12}$ will be

$C_{12}=\sqrt{1-t_{\mathrm{f}}^{2}} \operatorname{sgn}\left(G_{1}\right) \operatorname{sgn}\left(G_{2}\right)$.

When the two voltages $E_{\mathrm{n} 1}$ and $E_{\mathrm{n} 2}$ are subtracted in the second stage in Fig. 1, the output rms voltage noise $E_{\text {no }}$ can be computed from the output noise power [13],

$E_{\mathrm{n} 0}^{2}=E_{\mathrm{n} 1}^{2}+E_{\mathrm{n} 2}^{2}-2 C_{12} E_{\mathrm{n} 1} E_{\mathrm{n} 2}$.

Provided that $G_{1}$ and $G_{2}$ are both positive or negative, if the two signal bandwidths are identical $\left(t_{\mathrm{f}}=0\right)$, then $C_{12}=+1$. Nevertheless, mismatches as large as $f_{1}=2 f_{2}\left(t_{\mathrm{f}}=1 / 3\right)$ yield $C_{12}=0.94$, which is a good correlation. Therefore, because most differential amplifiers are expected to achieve high $C_{12}$ values, common-mode random noise $e_{\mathrm{ni}}$ in Fig. 1 should usually be largely cancelled.

\subsection{Common-mode electronic noise reduction factor}

Substituting (6)-(10) in (12) yields

$$
\begin{aligned}
E_{\mathrm{no}}^{2} & =\pi e_{\mathrm{ni}}^{2} G_{1}^{2} f_{1}+\pi e_{\mathrm{ni}}^{2} G_{2}^{2} f_{2}-4 \frac{f_{1} f_{2}}{f_{1}+f_{2}} \pi e_{\mathrm{ni}}^{2} G_{1} G_{2} \\
& =\pi e_{\mathrm{ni}}^{2}\left(G_{1}^{2} f_{1}+G_{2}^{2} f_{2}\right)\left[1-4 \frac{G_{1} G_{2}}{\left(G_{1}^{2} f_{1}+G_{2}^{2} f_{2}\right)} \frac{f_{1} f_{2}}{\left(f_{1}+f_{2}\right)}\right] \\
& =E_{\mathrm{u}}^{2}\left(1-k_{\mathrm{NR}}\right)
\end{aligned}
$$

where 
$E_{\mathrm{u}}^{2}=\pi e_{\mathrm{ni}}^{2}\left(G_{1}^{2} f_{1}+G_{2}^{2} f_{2}\right)$

is the noise power obtained when $e_{\mathrm{n} 1}(f)$ and $e_{\mathrm{n} 2}(f)$ are totally uncorrelated, and

$k_{\mathrm{NR}}=4 \frac{G_{1} G_{2}}{G_{1}^{2} f_{1}+G_{2}^{2} f_{2}} \frac{f_{1} f_{2}}{f_{1}+f_{2}}=\frac{4}{\frac{G_{1}}{G_{2}}+\frac{G_{2}}{G_{1}}+\frac{G_{1} f_{1}}{G_{2} f_{2}}+\frac{G_{2} f_{2}}{G_{1} f_{1}}}$

is a factor that describes the amount of noise reduction with respect to the case with uncorrelated noise in the two signal paths, and will always be positive.

Gains can also be written in terms of their tolerance. Using analogous definitions to $t_{\mathrm{f}}$, we rewrite $G_{1}=G_{\mathrm{a}}\left(1+t_{\mathrm{G}}\right)$ and $G_{2}=G_{\mathrm{a}}(1-$ $\left.t_{\mathrm{G}}\right)$. Substituting all gains and bandwidths in terms of their tolerances yields

$k_{\mathrm{NR}}=\frac{1-t_{\mathrm{G}}^{2}}{1+\left(t_{\mathrm{G}}+t_{\mathrm{f}}\right)^{2} /\left(1-t_{\mathrm{f}}^{2}\right)}$.

Perfect bandwidth matching $\left(t_{f}=0\right)$ yields

$\left.k_{\mathrm{NR}}\right|_{t_{\mathrm{f}}=0}=\frac{1-t_{\mathrm{G}}^{2}}{1+t_{\mathrm{G}}^{2}} \approx 1-2 t_{\mathrm{G}}^{2}$

for $t_{\mathrm{G}} \ll 1$, while perfect gain matching $\left(t_{G}=0\right)$ yields

$\left.k_{\mathrm{NR}}\right|_{t_{\mathrm{G}}=0}=1-t_{\mathrm{f}}^{2}$.

Perfect gain-bandwidth product (GBW) matching, i.e. $G_{1} f_{1}=G_{2} f_{2}$, which implies $t_{\mathrm{f}}=-t_{\mathrm{G}}$ (mismatches equal in magnitude but of opposite sign), yields

$\left.k_{\mathrm{NR}}\right|_{t_{\mathrm{G}}=0}=1-t_{\mathrm{G}}^{2}$

Ideal conditions $\left(t_{f}=t_{G}=0\right)$ yield $k_{\mathrm{NR}}=1$ and the input commonmode voltage noise would not contribute any output voltage noise. This situation will seldom be achieved in practice but, according to Fig. 2, more realistic tolerance values already yield high $k_{\mathrm{NR}}$ values hence common-mode voltage noise will often be largely cancelled. If we assume, for example, $\pm 20 \%$ manufacturing tolerances $\left(t_{G}=t_{f}=0.2\right.$ ) we obtain $C_{12}=0.98$ and $k_{\mathrm{NR}}=0.82$, which is quite good. Similarly, $\pm 5 \%$ tolerances yield $C_{12}=0.99875$ and $k_{\mathrm{NR}}=0.9975$, which is excellent. This implies that common-mode electronic noise will seldom be a problem, as usually assumed, and that $k_{\mathrm{NR}}$ is more sensitive to mismatches than $C_{12}$ hence better describes them.

\subsection{CMRR and Common-mode noise reduction}

The limited CMRR for deterministic signals in differential circuits also results from component or circuit mismatches and, as opposed to the factor $k_{\mathrm{NR}}$ above, it is normally specified for differ- ential amplifiers. Therefore, if there were any equation that related them, perhaps CMRR could be used to predict common-mode electronic noise reduction.

For a differential circuit like that in Fig. 1, where the two signal paths in the first stage are independent from each other, according to $[14,15]$ we have,

$\operatorname{CMRR}(f)=\frac{1}{2} \frac{H_{1}(f)+H_{2}(f)}{H_{1}(f)-H_{2}(f)}$

Therefore, whereas for signal cross-correlation, circuit bandwidth matching is evaluated in (10) by the quotient between their geometric and arithmetic means, and this quotient will be close to 1 even for relatively large bandwidth mismatches, for the CMRR the average of the two transfer functions is compared to their difference and the result will be small whenever the two transfer functions are not very close.

Replacing (1) and (2) in (16) yields

$\operatorname{CMRR}(f)=\frac{1}{2} \frac{\left(G_{1}+G_{2}\right) f_{1} f_{2}+j f\left(G_{1} f_{1}+G_{2} f_{2}\right)}{\left(G_{1}-G_{2}\right) f_{1} f_{2}+j f\left(G_{1} f_{1}-G_{2} f_{2}\right)}$

that is a complex parameter whereas $k_{\mathrm{NR}}$ is a real number. A perfect CMRR requires $G_{1}=G_{2}$ and $G_{1} f_{1}=G_{2} f_{2}$, which are the same conditions that yield $k_{\mathrm{NR}}=1$. If $G_{1} \neq G_{2}$, then at dc,

$\operatorname{CMRR}(0)=\frac{1}{2 t_{\mathrm{G}}}$

whereas at very high frequencies,

$\operatorname{CMRR}(\infty)=\frac{1+4 t_{\mathrm{G}} t_{\mathrm{f}}}{2 t_{\mathrm{G}}+2 t_{\mathrm{f}}} \simeq \frac{1}{2\left(t_{\mathrm{G}}+t_{\mathrm{f}}\right)}$.

That is, close gains improve $\operatorname{CMRR}(0)$, and $k_{\mathrm{NR}}$, whereas close bandwidths make $\operatorname{CMRR}(f) \approx \operatorname{CMRR}(0)$, hence constant with frequency but not necessarily high (for example if dc gains are unbalanced) yet improve $C_{12}$ hence $k_{\mathrm{NR}}$. If gains and bandwidth are mismatched but GBW is matched then, from (19),

$k_{\mathrm{NR}}=1-\frac{1}{4 \times \operatorname{CMRR}(0)^{2}}$.

Therefore, $k_{\mathrm{NR}}$ will always be very close to 1 in this case.

These results demonstrate that $k_{\mathrm{NR}}$ is far less sensitive to mismatching than the CMRR. Thus, for the same example above with $\pm 20 \%$ tolerance for dc gain and signal bandwidth, we obtain $\operatorname{CMRR}(0)=2.5(8 \mathrm{~dB})$, which would not significantly reduce input common-mode voltages, often much larger than differential signals, yet $k_{\mathrm{NR}}=0.82$ could be acceptable as common-mode noise is not usually as large as common-mode voltage interference can be.
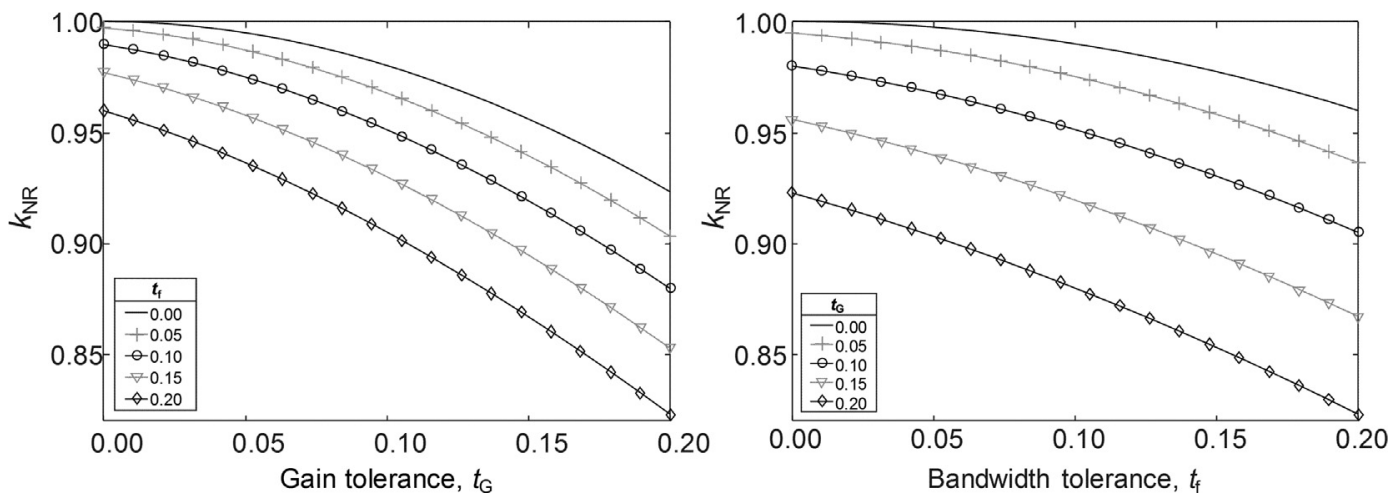

Fig. 2. Common-mode noise reduction factor $\left(k_{\mathrm{NR}}\right)$ as a function of gain tolerance (left) and of bandwidth tolerance (right) 
If the spectrum of $e_{\text {ni }}(f)$ were colored, for example because it includes $1 / f$ noise, the equations (10), (13) and (15) -for $C_{12}, E_{\text {no }}^{2}$ and $k_{\mathrm{NR}}$ respectively- would not apply. Nevertheless, the conclusion that differential circuits largely reject input common-mode electronic noise even if gain and signal bandwidth are so poorly matched that the CMRR is low, can be expected to remain true. The same can be said if the frequency response of the circuit is band-pass instead of low-pass, or is second order instead of first order.

Nevertheless, it would be wrong to draw the conclusion that a large CMRR will necessarily imply a large $k_{\mathrm{NR}}$, because the result above applies only to differential circuits that can be modelled by Fig. 1 . The differential circuit in Fig. 3 (left), for example, comprises an unbalanced fully-differential low-pass filter connected to a differential amplifier. Since there is no return path for common-mode currents, the filter's CMRR will be infinite and $V_{\mathrm{cm}}$ will not contribute to the output voltage. However, the input common-mode voltage noise $e_{\mathrm{ni}}$ will propagate differently to the inverting and non-inverting inputs (Fig. 3, right) yielding a low $C_{12}$, hence a low $k_{\mathrm{NR}}$, and it will contribute to the output voltage noise.

In summary, even though no simple equation relates $k_{\mathrm{NR}}$ and $\mathrm{CMRR}$, and $k_{\mathrm{NR}}$ cannot be directly calculated from data sheet specifications, neither a large CMRR necessarily means a high $k_{\mathrm{NR}}$, input common-mode electronic noise in balanced differential circuits can usually be neglected because of the subtraction included in differential signal processing.

\subsection{Implications of common-mode noise reduction in integrated differential amplifiers}

The first consequence of the great ability of differential circuits of rejecting input common-mode electronic noise even if their CMRR is small is that, for optimal noise performance, any passive front-end for differential circuits should be so designed that differential noise is reduced disregarding possible common-mode noise increases, because this last noise will be rejected. Differential passive front-end circuits may be required, for example, for ac-coupling or to provide a biasing path for the input stages of a differential circuit connected to a capacitive signal source or to a floating signal source [16-18], like that shown in Fig. 4. Here, $Z_{\mathrm{i}}$ and $Z_{\mathrm{i}}$ ' should be large-enough to achieve the desired differential input impedance, but not larger, whereas $Z_{\mathrm{b}}$ can include a much larger resistor, commensurate to the input bias current and the acceptable input offset voltage. Large resistors in $Z_{\mathrm{i}}$ and $Z_{\mathrm{i}}{ }^{\prime}$ would yield differential-mode noise (thermal noise and voltage noise due to equivalent input current noise sources of each input terminal, $i_{\mathrm{n}+}$ for the non-inverting input and $i_{\mathrm{n}-}$ for the inverting input), whereas $Z_{\mathrm{b}}$ will produce only common-mode noise.

The second major consequence of the result of the analysis above relates to $i_{\mathrm{n}^{+}}$and $i_{\mathrm{n} \text {. }}$. Whenever these noise sources are

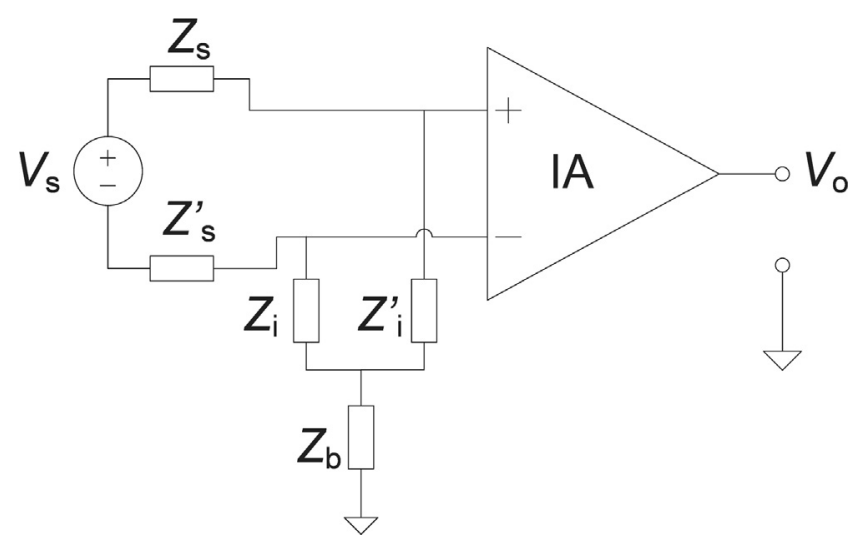

Fig. 4. Connecting a floating signal source $V_{s}$ to the inputs of an instrumentation amplifier (IA) that has a different signal ground requires an input biasing network for the amplifier.

highly correlated, most of the voltage they develop across the equivalent source resistors will be common-mode noise hence output noise will be smaller than that resulting from a straight rms sum (for uncorrelated noise sources). For operational amplifiers, for example, it has been demonstrated in [19] that a complete noise model requires an equivalent input voltage noise generator, $e_{\mathrm{n}}$, and $i_{\mathrm{n}^{+}}=i_{\mathrm{n}-}=i_{\mathrm{n}}$ and the three corresponding correlation coefficients between each pair of these three generators. The measured correlation between voltage and current sources in a single op amp in that study was about 0.05 at low frequencies and 0.02 at high frequencies whereas the (negative) correlation between $i_{n^{+}}$and $i_{\mathrm{n} \text { - }}$ was about 0.5 and constant with frequency. In [20], however, only the correlation between the voltage noise source and each of the two current noise sources was considered, and the result was a negative correlation that was stronger at frequencies where the $1 / f$ noise predominates. Correlation between $i_{\mathrm{n}^{+}}$and $i_{\mathrm{n}-}$ was disregarded because they are connected to different input terminals. In [19] correlation between all noise sources was considered "because they may include the contribution of the same noise physical mechanism." As noted in [11], some op amp topologies like bipolar input op amps have an emitter biasing circuitry whose noise yields correlated input current noise on both input bipolar transistors. Current noise in other technologies like CMOS op amps are due to the reversed biased input diodes that are physically different hence their noise will be totally uncorrelated. The analysis in Section II.A offers a complementary point of view: equivalent input noise generators will produce differential-mode and commonmode voltages and only differential voltages will contribute to the output voltage noise. Whenever there is significant correlation between $i_{n+}$ and $i_{n-}$ the common-mode voltage will increase for matched source resistors and this is why the specifications of some low-noise op amps (e.g. LT1028 from Linear Technology) apply
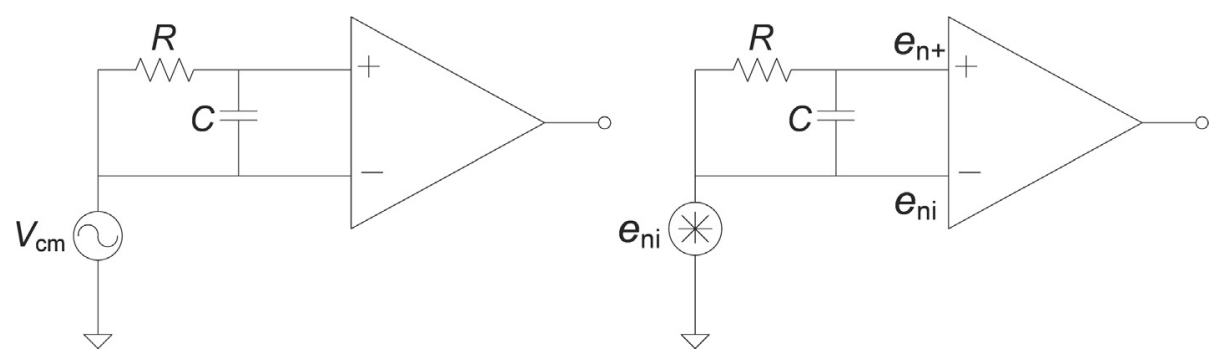

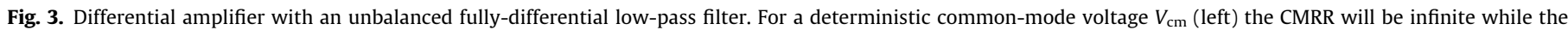

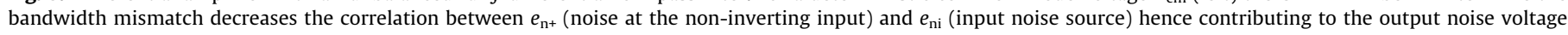
(right). 
only for matched source resistors. This could also explain why the maximal noise overestimation in [19] was obtained for matched source resistors.

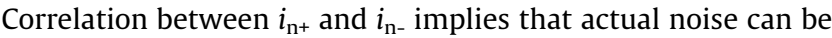
smaller than predicted noise, as data sheets do not usually include any correlation coefficients. Anyway, lower noise is an advantage hence this is not necessarily an issue. However, if the instrumentation amplifier in Fig. 4 is replaced by a differential amplifier built

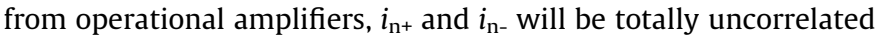
hence they will contribute differential noise.

\section{Noise measurement setup}

The common-mode noise rejection predicted by (13), the low relevance of the CMRR in noise calculations, and the correlation between equivalent input current noise sources in integrated IAs have been assessed by two different experiments.

First, noise predictions based on (13) and the role of the CMRR have been evaluated by measuring the output voltage noise of a differential amplifier built to resemble the circuit model in Fig. 1 as shown in Fig. 5. $G_{1}$ was the gain of a non-inverting amplifier, $G_{2}$ was the gain of an inverting amplifier, and the summing stage was a voltage adder, all built from op amps. Although $G_{2}$ was negative, the adder made this setup equivalent to the differential-tosingle-ended conversion circuit modelled in Fig. 1 hence the analysis above applies if $G_{2}$ is assumed positive. This circuit allows us to easily imbalance gains and has slightly different signal bandwidths for each signal path even when $G_{1}=G_{2}$ because for the non-inverting amplifier $f_{1}=\mathrm{GBW}_{1} / G_{1}$ whereas for the inverting amplifier $f_{2}=\mathrm{GBW}_{2} /\left(1+\left|G_{2}\right|\right)$ [21]. This imbalance, however, decreases for increasing gains. First, three different sets of close values for $G_{1}$ and $G_{2}$ were selected, around 10 (case 1), 40 (case 2 ) and 60 (case 3). Later, in order to assess the effect of a very poor CMRR, gains were designed $G_{1}=1$ and $\left|G_{2}\right|=10$ (case 4), which from (22) yield $\operatorname{CMRR}(0)=0.6$. The experimental tolerances $t_{G}$ and $t_{f}$ are shown in Table 1.

All op amps used were OP270 (Analog Devices) with $\mathrm{GBW}=5 \mathrm{MHz}$, typical. The external input voltage noise $E_{\mathrm{ni}}$ was obtained from a function generator (33210A, Agilent Technologies) with rms values from $100 \mathrm{mV}$ (for voltage gains larger than 10) and $2 \mathrm{~V}$ (for voltage gains 10 and smaller), selected to have a largeenough output voltage that could be easily measured. All rms noise voltages were measured with the AC rms voltage (standard deviation) measurement function of a digital oscilloscope (DSO-X 3254A, Agilent Technologies). The equivalent noise bandwidth was from $0.1 \mathrm{~Hz}$ to $100 \mathrm{kHz}$ which resulted from acquiring $10 \mathrm{~s}$ time series at $200 \mathrm{kHz}$ sampling frequency (See Fig. 6).

In a second scenario, we measured the output voltage noise due to a large-value resistor $R_{\mathrm{b}}$ connected to the input of an IA in differ-

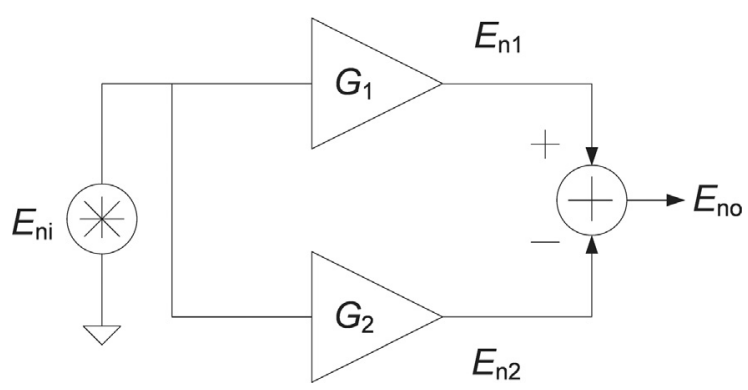

Fig. 5. Differential amplifier built from separated inverting and non-inverting amplifiers and a summing amplifier that performs the differential-to-single-ended signal conversion. $E_{\mathrm{ni}}$ is the rms value of a random voltage from a signal generator that is simultaneously applied to both inputs. ent ways (Fig. 7). The value of $R_{\mathrm{b}}$ was chosen high enough to make the voltage drop across it due to $i_{\mathrm{n}+}$ and $i_{\mathrm{n} \text { - }}$ to dominate over $e_{\mathrm{n}}$ and the own resistor's thermal noise. Case a) allows us to measure the rms noise floor, $E_{\mathrm{n}} . R_{\mathrm{b}}$ in cases $\mathrm{b}$ ), c) and d) yields differential-mode noise only and $E_{\mathrm{nd}}$ should match the rms sum of $E_{\mathrm{nb}}$ and $E_{\mathrm{nc}}$ if input noise current sources were uncorrelated,

$E_{\mathrm{nd}}=\sqrt{E_{\mathrm{nb}}^{2}+E_{\mathrm{nc}}^{2}}$.

The equivalent common-mode input voltage noise at the inverting and non-inverting inputs in case e) is

$e_{\mathrm{n}+}^{2}=e_{\mathrm{n}-}^{2} \approx\left(i_{\mathrm{n}+}^{2}+i_{\mathrm{n}-}^{2}\right) R_{\mathrm{b}}^{2}$

which should be largely rejected so that the output voltage noise should be close to $E_{n}$.

In this second set of experiments, the output voltage noise was measured with a $61 / 2$ digit voltmeter (34401A, Agilent Technologies) by computing the standard deviation of 100 consecutive measurements (dc voltage, 10 power-line cycle integration time), which results in $5 \mathrm{~Hz}$ measurement bandwidth and negligible internal noise (Fig. 8). The commercial monolithic IAs tested were INA114 (Texas Instruments) and AD623 (Analog Devices). The specific setup for each IA is described in Table 2.

\section{Experimental results and discussion}

Table 1 shows the results for the differential amplifier in Fig. 5. $G_{1}$ and $G_{2}$ are the actual gains whereas $\operatorname{CMRR}(0)$ has been calculated from (18). $f_{1}$ and $f_{2}$ have been calculated from the op amp's gain-bandwidth product, and $C_{12}$ and $k_{\mathrm{NR}}$ are those predicted by (10) and (15) respectively. $E_{\mathrm{n} 1}$ and $E_{\mathrm{n} 2}$ are the measured output voltages for the signal paths with gain $G_{1}$ and $G_{2}$ respectively, $E_{\mathrm{u}}$ would be the expected output voltage if $E_{\mathrm{n} 1}$ and $E_{\mathrm{n} 2}$ were uncorrelated, $E_{\mathrm{no}, \mathrm{t}}$ is the output voltage predicted from (13), and $E_{\mathrm{no}, \mathrm{m}}$ is the measured output voltage, due to both $E_{\text {no,t }}$ and any equivalent differential input voltage noise, probably much smaller than the external noise applied in common mode.

For cases 1 to $3, t_{\mathrm{G}}$ and $t_{\mathrm{f}}$ are sufficiently low for the predicted values of $C_{12}$ and $k_{\mathrm{NR}}$ to approach their ideal values in spite of the relatively poor $\operatorname{CMRR}(0)$ in case 1 as compared to the usual $\operatorname{CMRR}(0)$ values required to reject common mode input signals, and of the mismatch between $G_{1} f_{1}$ and $G_{2} f_{2}$. Measured $E_{\mathrm{n} 1}$ and $E_{\mathrm{n} 2}$ voltages are similar in the three cases hence attributable to the noise voltage applied in common mode. If $E_{\mathrm{n} 1}$ and $E_{\mathrm{n} 2}$ were independent, the resulting output voltage when added, $E_{\mathrm{u}}$, would be very large. However, measured output voltages $E_{\mathrm{no}, \mathrm{m}}$ are very close to the calculated $E_{\mathrm{no}, \mathrm{t}}$ values, which corroborates that the actual $k_{\mathrm{NR}}$ is close enough to 1 for the random common-mode voltage applied to the input ( $100 \mathrm{mV}$ or $2 \mathrm{~V}$, depending on the gain) to yield only a very small output voltage. This is also in agreement with the predicted behavior in Fig. 3 .

The scant influence of a large $\operatorname{CMRR}(0)$ in common-mode noise reduction can be observed by comparing cases 1 and 2: in case 1 , voltage noise reduction with respect to $E_{\mathrm{u}}$ is about $95 \%(55.8 \mathrm{mV}$ compared to $1183.5 \mathrm{mV}$ ), whereas in case 2 a CMRR $(0)$ about seven times better increases voltage noise reduction only to $98.5 \%$ (36 mV compared to $2402.9 \mathrm{mV}$ ).

Case 4 worsens the dissimilitude between the two signal paths for the applied random common-mode voltage. The dissimilar gains result in $\operatorname{CMRR}(0)=0.6$ and the large signal bandwidth mismatch $(462 \mathrm{kHz}$ and $4824 \mathrm{kHz}$ ) predicts poor correlation (about 0.56 ) and power noise reduction, $k_{\mathrm{NR}}=0.33$. In practice, voltage noise reduces from $300 \mathrm{mV}$ to $251 \mathrm{mV}$, which is equivalent to $30 \%$ power noise reduction. Therefore, predictions from (13) are right. 
Table 1

Measured values for total output voltage for the differential amplifier in Fig. 5 when a random voltage Eni is applied in common mode.

\begin{tabular}{|c|c|c|c|c|c|c|c|c|c|c|}
\hline Case & $t_{\mathrm{G}}$ & $t_{\mathrm{f}}$ & CMRR(0) linear & $C_{12}$ & $k_{\mathrm{NR}}$ & $E_{\mathrm{n} 1} / \mathrm{mV}$ & $E_{\mathrm{n} 2} / \mathrm{mV}$ & $E_{\mathrm{u}} / \mathrm{mV}$ & $E_{\mathrm{no}, \mathrm{t}} / \mathrm{mV}$ & $E_{\mathrm{no}, \mathrm{m}} / \mathrm{mV}$ \\
\hline 1 & 0.046 & 0.002 & 11 & -0.99983 & 0.99719 & 861 & 812 & 1183.5 & 49.0 & 55.8 \\
\hline 2 & 0.007 & 0.006 & 70 & -0.99989 & 0.99989 & 1715 & 1683 & 2402.9 & 33.4 & 36.0 \\
\hline 3 & 0.008 & 0.016 & 63 & -0.99998 & 0.99977 & 287 & 281 & 401.7 & 7.5 & 7.2 \\
\hline 4 & 0.816 & 0.832 & 0.6 & -0.564880 & 0.33359 & 73 & 291 & 300.0 & 257.7 & 251.0 \\
\hline
\end{tabular}

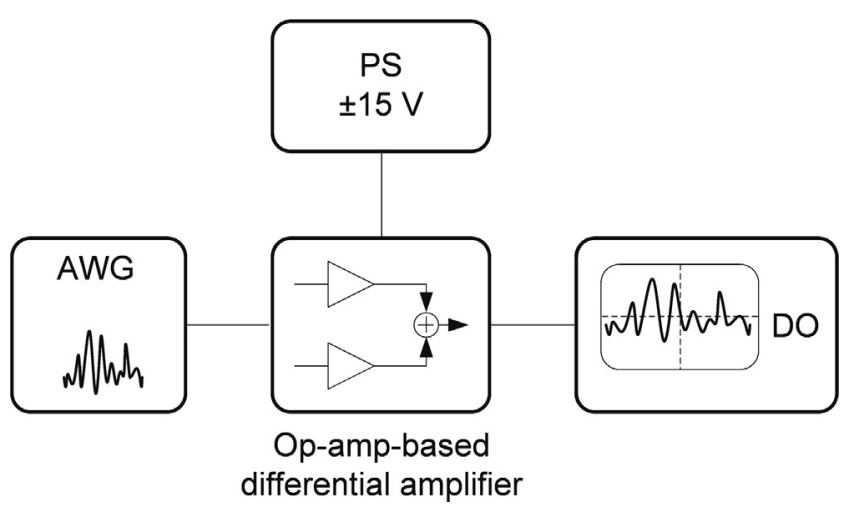

Fig. 6. Noise measurement setup for the differential amplifier built from spare op amps (Fig. 5). White noise is generated by an arbitrary waveform generator (AWG). The power supply (PS) provides $\pm 15 \mathrm{~V}$ to the op amp. Finally, a digital oscilloscope (DO) records the rms output voltage.

In monolithic IAs, spectral correlation and $\operatorname{CMRR}(f)$ will likely be high because of component trimming during manufacturing hence common-mode noise reduction should be large. Table 2 shows that noise in Fig. $7 \mathrm{~b}$ and $\mathrm{c}$ is about the same, as expected because $i_{\mathrm{n}+}$ and $i_{\mathrm{n}-}$ are assumed to be equal for both input terminals. Therefore, if $i_{\mathrm{n}+}$ and $i_{\mathrm{n}-}$ were uncorrelated, from (25) it could be expected that $E_{\text {nd }}$ in Fig. $7 \mathrm{~d}$ were about $3.65 \mathrm{mV}$, yet the measured $E_{\mathrm{nc}}$ is only $2.41 \mathrm{mV}$. This means that input noise currents have a strong negative correlation. As a matter of fact, applying (12) to $E_{\mathrm{nb}}, E_{\mathrm{nc}}$ and $E_{\mathrm{nd}}$ allows us to estimate a correlation factor of -0.57 between $i_{n^{+}}$and $i_{\text {n- }}$ for INA114 and -0.23 for AD627. This partial correlation was further corroborated by repeating the same test on ten INA114 and ten AD627 units. The calculated correlation factors were between -0.1 and -0.6 for the INA114 and also for the AD627.

In Fig. 7e, it is safe to assume $C_{12} \approx 1$ and the large CMRR(0) justifies the suppression of common-mode noise hence $E_{\text {ne }}$ should in principle be a bit larger than $E_{\mathrm{n}}$ (noise floor). However, whereas $E_{\mathrm{ne}}$ is certainly more than two orders of magnitude smaller than $E_{\mathrm{nd}}$, it turns out that for the AD627, $E_{\mathrm{ne}}<E_{\mathrm{n}}$, which suggests that crosscorrelations between its intrinsic noise sources $e_{\mathrm{n}}, i_{\mathrm{n}+}$ and $i_{\mathrm{n}-}$ are stronger than for the INA114. In any case, the reduction of input common-mode noise contribution to output voltage noise in differential amplifiers is undisputable and the circuits in Fig. $7 \mathrm{~d}$ and 7e provide a fast method to evaluate it. Further, whereas in Fig. 5 the input common-mode noise was an external random signal with white power spectral density, the common-mode noise in Fig. 7 e includes $1 / f$ noise from $e_{\mathrm{n}}, i_{\mathrm{n}^{+}}$and $i_{\mathrm{n} \text { - yet the large beneficial }}$ effect of the internal voltage subtraction in differential amplifiers is also observed, as expected. Therefore, when designing passive front ends such as input biasing networks [22] or ac-coupling filters [23], that can be modelled as in Fig. 4 with a common-mode

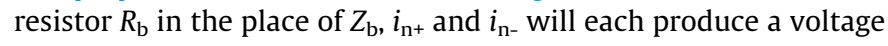

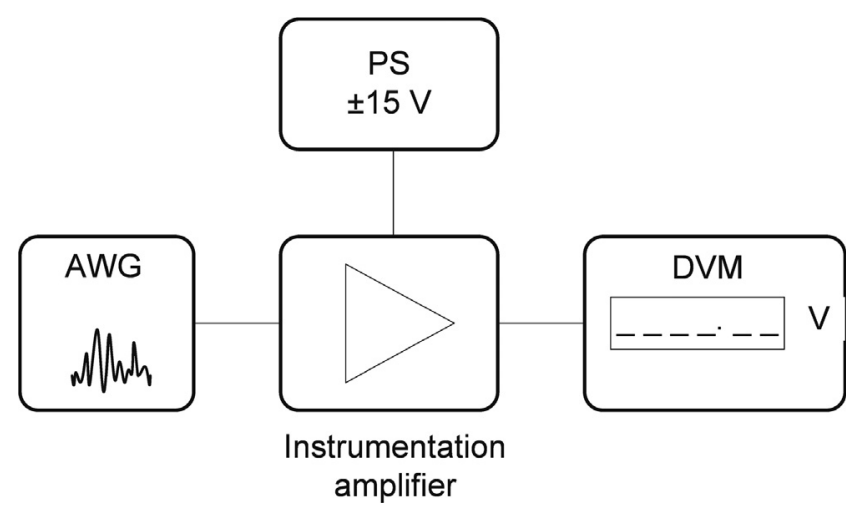

Fig. 8. Noise measurement setup for the different instrumentation amplifier configurations of Fig. 7. The instruments used are the same as in Fig. 6 except for the DO that has been replaced by a $61 \frac{1}{2}$ digital voltmeter (DVM).

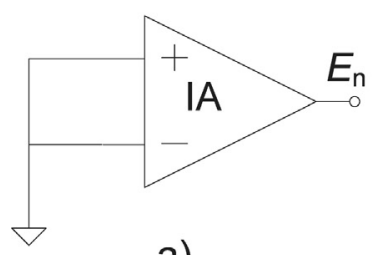

a)

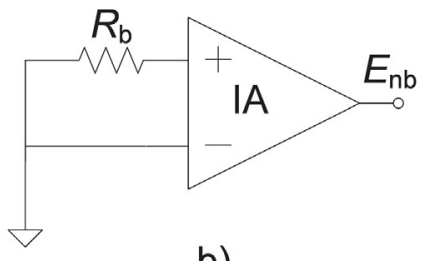

b)

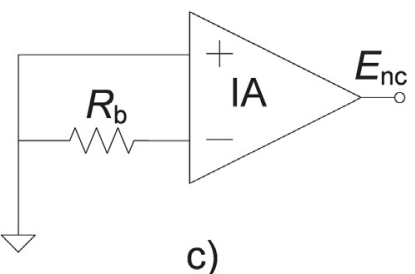

c)

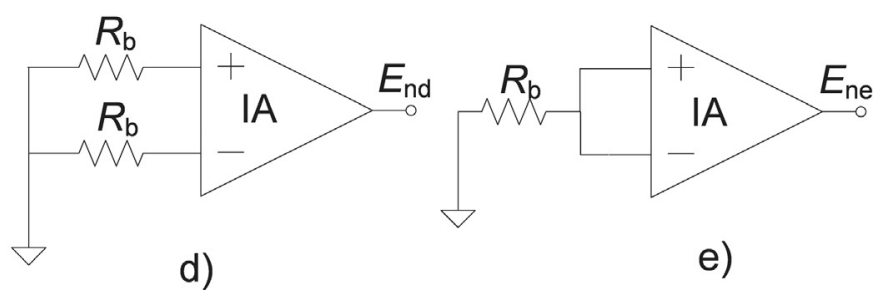

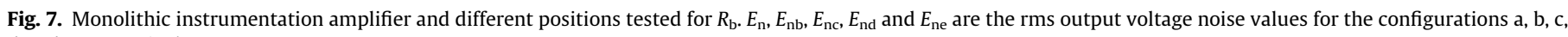
$\mathrm{d}$ and e respectively. 
Table 2

Measured values for total output noise for the different circuits in Fig. 7.

\begin{tabular}{|c|c|c|c|c|c|c|c|c|}
\hline \multicolumn{4}{|c|}{ Instrumentation amplifier characteristics } & \multicolumn{5}{|c|}{ Measurements } \\
\hline Model & Setup & CMRR (0)/ dB Typ & Noise sources (@ 1 kHz) & $E_{\text {na }} / \mu \mathrm{V}$ & $E_{\mathrm{nb}} / \mathrm{mV}$ & $E_{\mathrm{nc}} / \mathrm{mV}$ & $E_{\text {nd }} / \mathrm{mV}$ & $E_{\mathrm{ne}} / \mu \mathrm{V}$ \\
\hline INA114 & $R_{\mathrm{b}}=10 \mathrm{M} \Omega G=10$ & 106 & $e_{\mathrm{n}}=11 \mathrm{nV} / \sqrt{ } \mathrm{Hz} i_{\mathrm{n}}=0,2 \mathrm{pA} / \sqrt{ } \mathrm{Hz} f_{\mathrm{ci}}>10 \mathrm{~Hz}$ & 6.10 & 2.45 & 2.70 & 2.41 & 6.98 \\
\hline AD627 & $R_{\mathrm{b}}=55 \mathrm{M} \Omega G=45$ & 100 & $e_{\mathrm{n}}=38 \mathrm{nV} / \sqrt{ } \mathrm{Hz} i_{\mathrm{n}}=50 \mathrm{fA} / \sqrt{ } \mathrm{Hz} f_{\mathrm{ci}} \approx 5 \mathrm{~Hz}$ & 10.30 & 0.38 & 0.36 & 0.46 & 8.80 \\
\hline
\end{tabular}

noise, uncorrelated to $R_{\mathrm{b}}$ 's thermal noise, and the three noise voltages will appear in common mode hence they will be rejected.

The conclusion from the analysis of the circuit in Fig. 7e can be applied to the design of IAs built from discrete op amps, such as the three-op-amp instrumentation amplifier. Here, the equivalent input current noise sources will be uncorrelated hence an input biasing T-network like that in Fig. 4 will largely outperform a network consisting of a single biasing resistor from each amplifier input to signal ground but in any case the circuit will be noisier than a monolithic IA.

\section{Conclusions}

Input common-mode electronic noise in differential circuits is usually disregarded because of the common-mode rejection ratio (CMRR) thus omitting the fact that the CMRR is defined only for deterministic signals, not for random noise.

Input common-mode noise in differential circuits yields internal noise voltages that are eventually subtracted before contributing to the output voltage noise. That subtraction results in negative correlation between the output voltages of the two signal paths of the differential circuit and if these two paths were matched, then input common-mode noise would be totally rejected upon subtracting their outputs. Mismatches between the two signal paths, however, limit noise reduction according to factor $k_{\mathrm{NR}}$ defined in (15), which should ideally be 1 . High CMRR values for the entire signal bandwidth yield $k_{\mathrm{NR}} \approx 1$ and they can be obtained by high CMRR(0) and similar gain-bandwidth product for the two signal paths $\left(\mathrm{GBW}_{1} \approx \mathrm{GBW}_{2}\right)$, the first condition being more important. Nevertheless, a very high CMRR by itself does not guarantee common-mode noise reduction. Circuit topologies like that in Fig. 3 where there's no return path for common-mode currents might completely cancel the effects of common-mode voltage signals but not the contribution of common-mode random noise because unbalanced noise paths to the inverting and noninverting inputs yield partially uncorrelated signals that would eventually contribute output noise.

Experimental results in a differential amplifier built to enable controlled mismatching of the two signal paths confirm theoretical predictions and corroborate that both gain and bandwidth should be similar in order to decrease common-mode electronic noise. However, no strict matching is required because more than $95 \%$ noise reduction can be obtained with just $\operatorname{CMRR}(0)=11(21 \mathrm{~dB})$. These conditions are easy to achieve in monolithic differential amplifiers but require manual passive component trimming in differential circuits built from discrete components.

Monolithic differential (instrumentation) amplifiers have the additional advantage of the negative correlation between their input equivalent voltage and current noise sources and between both current noise sources, which reduces differential-mode noise. These advantages can be lost when the amplifier is preceded by a front-end with passive components, particularly high-value resistors, that can become the predominant noise sources due to their thermal noise and amplifier input noise currents. This could be the case, for example, of input biasing networks or ac-coupling filters. In these cases, large resistors must be placed in circuit positions where they contribute common-mode noise, as in Fig. 7e, rather than differential-mode noise, as in Fig. 7d. In other words, input biasing T-networks like that in Fig. 4 are preferred. Instrumentation amplifiers built from discrete components will always be noisier whatever the input biasing network because their input noise currents will be uncorrelated.

\section{Acknowledgments}

The authors thank the Castelldefels School of Telecommunications and Aerospace Engineering (EETAC-UPC) for its research facilities and Mr. F. López for his technical support. This research did not receive any specific grant from funding agencies in the public, commercial, or not-for-profit sectors.

\section{Appendix. . Derivation of (5)}

$$
\begin{aligned}
R_{12}(0)= & \frac{1}{2 \pi} \int_{-\infty}^{\infty} e_{\mathrm{n} 1}(f) e_{\mathrm{n} 2}^{*}(f) \mathrm{d} f \\
= & \frac{e_{\mathrm{ni}}^{2}}{2 \pi} \int_{-\infty}^{\infty} H_{1}(j f) H_{2}^{*}(j f) \mathrm{d} f \\
= & \frac{e_{\mathrm{ni}}^{2}}{2 \pi} \int_{-\infty}^{\infty} \frac{G_{1}}{1+j_{\frac{f}{f_{1}}}} \cdot \frac{G_{2}}{1-\frac{f}{f_{2}}} \mathrm{~d} f \\
= & \frac{e_{\mathrm{n}}^{2} G_{1} G_{2}}{2 \pi} \int_{-\infty}^{\infty}\left[\frac{1+\frac{f^{2}}{f_{1} f_{2}}}{\left(1+\frac{f^{2}}{f_{1}^{2}}\right)\left(1+\frac{f^{2}}{f_{2}^{2}}\right)}-j \frac{\frac{f}{f_{1}}-\frac{f}{f_{2}}}{\left(1+\frac{f^{2}}{f_{1}^{2}}\right)\left(1+\frac{f^{2}}{f_{2}^{2}}\right)}\right] \mathrm{d} f \\
= & e_{\mathrm{ni}}^{2} G_{1} G_{2}\left[2 \pi \frac{f_{1} f_{2}}{f_{1}+f_{2}}\left(\tan ^{-1} \frac{f}{f_{1}}+\tan ^{-1} \frac{f}{f_{2}}\right)\right]_{-\infty}^{\infty} \\
& -e_{\mathrm{ni}}^{2} G_{1} G_{2}\left[\frac{f_{1} f_{2}}{2\left(f_{1}+f_{2}\right)}\left(\log \left(f^{2}+f_{1}^{2}\right)-\log \left(f^{2}+f_{2}^{2}\right)\right)\right]_{-\infty}^{\infty} \\
= & 2 \pi e_{\mathrm{ni}}^{2} G_{1} G_{2} \frac{f_{1} f_{2}}{f_{1}+f_{2}}
\end{aligned}
$$

\section{References}

[1] G.T. Ong, P.K. Chan, A power-aware chopper-stabilized instrumentation amplifier for resistive wheatstone bridge sensors, IEEE Trans. Instrum. Meas. 63 (2014) 2253-2263, https://doi.org/10.1109/TIM.2014.2308992.

[2] J.L. Schmalzel, D.A. Rauth, Sensors and signal conditioning, IEEE Instrum. Meas. Mag. 8 (2005) 48-53, https://doi.org/10.1109/MIM.2005.1438844.

[3] M. Engin, T. Dalbasti, M. Güldüren, E. Davasli, E.Z. Engin, A prototype portable system for EEG measurements, Meas. J. Int. Meas. Confed. 40 (2007) 936-942, https://doi.org/10.1016/j.measurement.2006.10.018.

[4] Y. Hayashida, M. Hasegawa, A Suzuki, M. Shinagawa, Y. Kado, N. Haga, Radiated noise analysis via human body for intra-body communication, Meas. J. Int. Meas. Confed. 89 (2016) 159-165, https://doi.org/10.1016/j. measurement.2016.03.071.

[5] D. Liu, C. He, Q. Zhao, Z. Yang, Y. Hao, G. Yan, Digital signal processing for a micromachined vibratory gyroscope based on a three dimensional adaptive filter demodulator, Meas. J. Int. Meas. Confed. 50 (2014) 198-202, https://doi. org/10.1016/j.measurement.2013.12.025.

[6] B. Razavi, Design of Analog CMOS Integrated Circuits, 2nd ed., McGraw-Hill Higher Education, San Francisco, 2017.

[7] E.V. Ivanov, Switched-capacitor level-shifting technique with sampling noise reduction for rail-to-rail input range instrumentation amplifiers, IEEE Trans Circuits Syst. I Regul. Pap. 59 (2012) 2867-2880, https://doi.org/10.1109/ TCSI.2012.2206455.

[8] S. Heo, H. Ma, J. Song K. Park, E.H. Choi, JJ. Kim, F. Bien, 72 dB SNR, 240Hz frame rate readout IC with differential continuous-mode parallel architecture for larger touch-screen panel applications, IEEE Trans, Circuits Syst. I Regul. Pap. 63 (2016) 960-971, https://doi.org/10.1109/TCSI.2016.2553319.

[9] G. Erdi, Amplifier techniques for combining low noise, precision, and highspeed performance, IEEE J. Solid-State Circuits. 16 (1981) 653-661, https://doi. org/10.1109/JSSC.1981.1051658. 
[10] W.M. Leach, Fundamentals of Low-Noise Analog Circuit Design, Proc. IEEE. 82 (1994) 1515-1538, https://doi.org/10.1109/5.326411.

[11] S. Franco, Op amp input current noise, Electron. Des. News - Analog Bytes Blog. (2013). http://www.edn.com/electronics-blogs/analog-bytes/4416424/Opamp-input-current-noise- (accessed September 7, 2017).

[12] J.S. Bendat, A.G. Piersol, Random data: analysis and measurement procedures, 4th ed., John Wiley \& Sons, Hoboken, USA, 2011.

[13] C.D. Motchenbacher, J.A. Connelly, Low noise electronic system design, Wiley, New York, USA, 1993.

[14] Ó. Casas, R. Pallàs-Areny, Basics of analog differential filters, IEEE Trans. Instrum. Meas. 45 (1996) 275-279, https://doi.org/10.1109/19.481347.

[15] R. Pallàs-Areny, O. Casas, A hands-on approach to differential circuit measurements, Measurement 40 (2007) 8-14, https://doi.org/10.1016/j. measurement.2006.05.008.

[16] J. Ramos, R. Pallàs-Areny, M. Tresànchez, Multichannel front-end for low level instrumentation signals, Measurement 25 (1999) 41-46, https://doi.org/ 10.1016/S0263-2241(98)00065-7.

[17] A. Affanni, G. Chiorboli, Design and characterization of a real-time, wearable, endosomatic electrodermal system, Meas. J. Int. Meas. Confed. 75 (2015) 111121, https://doi.org/10.1016/j.measurement.2015.07.047.
[18] B. Babusiak, S. Borik, L. Balogova, Textile electrodes in capacitive signal sensing applications, Meas. J. Int. Meas. Confed. 114 (2018) 69-77, https://doi.org 10.1016/j.measurement.2017.09.024

[19] G. Giusi, F. Crupi, C. Pace, P. Magnone, Full model and characterization of noise in operational amplifier, IEEE Trans, Circuits Syst. I Regul. Pap. 56 (2009) 97102, https://doi.org/10.1109/TCSI.2008.927011.

[20] J. Xu, Y. Dai, D. Abbott, A complete operational amplifier noise model: analysis and measurement of correlation coefficient, IEEE Trans, Circuits Syst. Fundam. Theory Appl. 47 (2000) 420-424, https://doi.org/10.1109/81.841928.

[21] S. Franco, Design with operational amplifiers and analog integrated circuits, 4th ed., McGraw-Hill series in electrical and computer engineering, New York, USA, 2015.

[22] O. Casas, R. Pallàs-Areny, Optimal bias circuit for instrumentation amplifiers, in: Proc. XIV Imeko World Congr., 1997: pp. 143-148.

[23] O. Casas, E.M. Spinelli, R. Pallàs-Areny, Fully Differential AC-Coupling Networks: A Comparative Study, IEEE Trans. Instrum. Meas. 58 (2009) 9498, https://doi.org/10.1109/TIM.2008.927200. 